\title{
Estudios Clínicos Controlados Aleatoreos, en la Pediatría de Latino América (1996-2005)
}

\author{
CARLOS CASTILlO D. ${ }^{1,2}$, XIMENA PLETIKOSIC C. ${ }^{1}$, FERNANDO PIZARRO A. ${ }^{1}$ \\ 1. Departamento de Pediatría, Facultad de Medicina Campus Centro, Universidad de Chile. \\ 2. Instituto de Nutrición y Tecnología de los Alimentos (INTA), Universidad de Chile.
}

\begin{abstract}
Randomized Controlled Clinical Studies in Latin American Pediatrics

Objective: To analyze the production of RCS among children and adolescents in Latin American countries, between 1996 and 2005. Method: In an observational bibliometric study, all available RCS in PUBMED and LILAC data bases for that period were reviewed. Included were studies of children between 0 and 18 years of age, from 19 Latin American countries; 400 references were included. As a control, all pediatric research from the State of New York, USA, for that same period of time was collected. The following parameters were evaluated: number of RCS per country, affiliation, pediatric area, and adjustments were made for population and GNP for each country. Results: Countries with the highest number of publications per year (RCS/yr) were: Brazil (14.6), Mexico (8.6), Chile (5.5), Argentina (3.9). During that same period of time, the State of New York published 26.7 RCS per year. Overall, in Latin America most published specialities were Nutrition (16\%), Respiratory Diseases, Dental problems, Vaccinations, Neonatology. In New York, the most frequent specialty reviewed was Psychiatry/Psychology (23.6\%). In Chile and Brazil, more than $85 \%$ of the studies were performed in Universities, in Mexico and Argentina in Hospitals and other organizations either public or private. Over $90 \%$ were published in Journals with ISI impact index. Conclusions: Random studies are rare in Latin America compared to developed countries, mostly conducted in Universities in few countries and focusing on pediatric topics of relevance to them.
\end{abstract}

(Key words: Research report, controlled, randomized, pediatrics, Latin America).

Rev Chil Pediatr 2009; 80 (5): 420-426

\section{RESUMEN}

Los estudios controlados aleatoreos (ECR) están entre los métodos más adecuados para evaluar la efectividad de intervenciones en Salud, pero hay grandes dificultades para efectuar este tipo de investigaciones en Latino América. Objetivo: Analizar la producción de ECR en países de Latino América en niños o adolescentes, en el período 1996-2005. Métodos: En un estudio observacional utilizando análisis bibliométrico, revisamos todos los ECR disponibles en las bases de datos PUBMED y LILAC entre 1996-

Trabajo recibido el 04 de abril de 2009, devuelto para corregir el 02 de julio de 2009, segunda versión el 08 de julio de 2009 , aceptado para publicación el 15 de julio de 2009.

Correspondencia a:

Dr. Carlos Castillo D.

E-mail: ccastd@med.uchile.cl 
2005. Se consideró estudios con niños entre 0 y 18 años, de 19 países Latinoamericanos; 400 referencias completaron los criterios. La investigación pediátrica del estado de New York, USA sirvió como control. Se evaluó: número de ECR por país, lugar de afiliación, área pediátrica, ajustes por tamaño de población del país y producto doméstico bruto. Resultados: Los países con más publicaciones fueron (ECR/año) fueron: Brasil $(14,6)$, México $(8,6)$, Chile $(5,5)$ y Argentina $(3,9)$. Durante el mismo período, el estado de New York publicó 26,7 ECR /año. En conjunto en LA, las especialidades pediátricas con más publicaciones fueron: nutrición (16\%), enfermedades respiratorias, dental, vacunas y neonatología. En New York, la especialidad más frecuente fue psiquiatría/psicología (23,6\%). En Chile y Brasil más del 85\% fueron efectuados por Universidades, en México y Argentina, fue más frecuente por hospitales y por otras organizaciones públicas o privadas. Sobre el $90 \%$ fueron publicadas en revistas con índice de impacto. Conclusiones: La proporción de estudios controlados aleatoreos en Latino América es baja, en comparación a países desarrollados; ellos fueron más frecuentemente efectuados por universidades, en temas pediátricos relevantes para la región y en unos pocos países.

(Palabras clave: Artículos, controlados, aleatorizados, Pediatría, Latinoamérica).

Rev Chil Pediatr 2009; 80 (5): 420-426

\section{Introducción}

Los estudios controlados aleatoreos (ECR) y las revisiones sistemáticas de ECR constituyen las formas más contundentes de evidencia clínica. Ellos son los mejores métodos de investigación clínica y proporcionan la base, a los clínicos para efectuar recomendaciones en temas específicos, a los administradores en salud y a los que trabajan con políticas en salud ${ }^{1,2}$.

Las publicaciones científicas en tópicos de salud humana están desproporcionadamente distribuidas y fuertemente concentradas en los países más desarrollados ${ }^{3}$. Los Estados Unidos de Norteamérica y la Unión Europea son las comunidades líderes en investigación biomédica y en publicaciones científicas ${ }^{4-6}$.

La investigación ha tenido poca prioridad en las políticas de salud en Latinoamérica (LA); hay en los países de la región pocos grupos de investigación establecidos y el financiamiento local es escaso o nulo ${ }^{7-12}$.

Además, en la investigación en salud humana se requieren diseños de investigación de mayor complejidad. Como consecuencia de todos estos hechos, la mayor parte del trabajo científico latinoamericano está limitado a casos clínicos o series clínicas, con muy pocos estudios controlados aleatorizados, controlados abiertos, de casos y controles o de cohorte. No conocemos de información acerca de ECR en Latinoamérica en años recientes.
Previamente se había analizado la investigación pediátrica en Chile ${ }^{13}$. El objetivo de esta investigación fue describir y analizar los ECR efectuados en población pediátrica latinoamericana, con la participación de investigadores locales, en el período 1996-2005. Adicionalmente analizamos esta investigación por países $y$ en relación a la efectuada en el estado de New York, USA.

\section{Métodos}

Se utilizó la base de datos PUBMED para identificar los ECR que incluyeron niños entre 0 y 18 años, publicados entre 1996 y 2005 y que fueron efectuados por instituciones y/o investigadores de 19 países latinoamericanos, a saber: Argentina, Brasil, Bolivia, Chile, Colombia, Costa Rica, Cuba, República Dominicana, Ecuador, El Salvador, Guatemala, Honduras, México, Nicaragua, Panamá, Paraguay, Perú, Uruguay y Venezuela. Usamos el nombre del país en la forma como está descrito en el campo de afiliación de PUBMED. El antecedente de ECR se analizó utilizando el botón de selección específico de la base de datos; si el resumen o abstract no especificaba la condición de ECR accedíamos al trabajo completo para evaluar esta condición. Para asegurarnos de la calidad de esta selección analizamos algunas de las revistas pediátricas de corriente principal, corroborando 
la calidad de esa selección para trabajos en revistas con índice de impacto ISI.

Identificamos los ECR con afiliación a instituciones o investigadores del estado de New York, como modelo representativo de un país desarrollado.

Además investigamos en la base de datos LILACS (Literature Latino Americana y del Caribe en Ciencias de la Salud), para ECR publicados en revistas científicas latinoamericanas no indexadas; también evaluamos la calidad de la selección revisando todos los artículos publicados en el período estudiado en las revistas pediátricas chilenas principales.

Se incluyó estudios perinatales que evaluaran resultados en neonatos, también se consideró estudios que incluyeran niños o adolescentes junto a adultos. Para comparar las investigaciones de acuerdo al tamaño del país, usamos la información en línea del Banco Mundial ${ }^{14}$, respecto de población y producto doméstico grueso bruto por país.

Se registró los siguientes datos de cada publicación: fecha de publicación, nombre de la revista científica, autores y afiliación, lugar de publicación y área de la Pediatría o sub-especialidad. Para aquellas publicaciones con más de un tema pediátrico o más de un centro investigador lo asignamos según área temática de la revista donde se publicó, o por especiali-

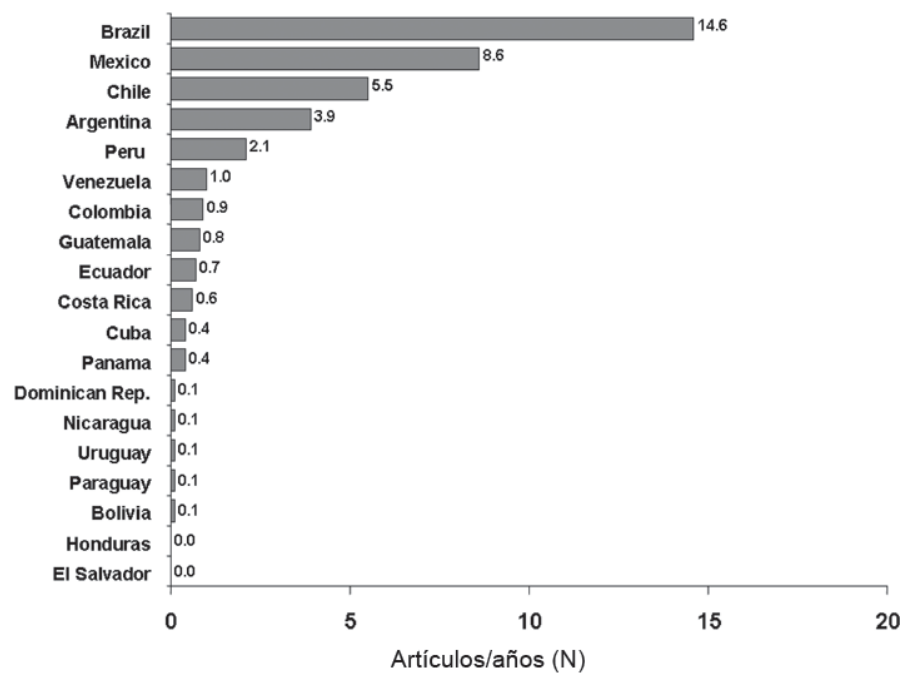

Figura 1. Estudios controlados aleatoreos en Pediatría, efectuados por países de Latino América (publicaciones/año, 1996-2005). dad del primer autor (determinada por sus publicaciones previas y afiliación a un centro especializado).

Las instituciones donde se efectuó la investigación fueron clasificadas como: Universidad, Hospital u otros (fundaciones, compañías farmacéuticas, asociaciones profesionales). Los hospitales universitarios fueron incluidos en la categoría de Universidad.

El análisis estadístico incluyó análisis de varianza (ANOVA), test post-hoc de Scheffé, y análisis of covarianza (ANCOVA) (Statistica 6.0, StatSoft Inc, Tulsa, OK).

\section{Resultados}

\section{Número de ECR y tendencias en la década}

Entre las publicaciones que cumplían con los criterios establecidos, encontramos 400 ECR en países Latino Americanos y 267 en el estado de New York. Los países que publicaron la mayor cantidad de publicaciones (trabajos/año) entre 1996 y 2005 fueron: Brasil $(14,6)$, México $(8,6)$, Chile $(5,5)$ y Argentina $(3,9)$; estos 4 países produjeron el $81 \%$ de los ECR pediátricos en Latino América (figura 1). En conjunto estos países produjeron 32,6 artículos/año y los restantes 7,4 artículos/año (ANOVA, F:8.46, $\mathrm{p}<0,0001)$. El test post-hoc de Scheffé mostró diferencias entre Brasil y Argentina $(\mathrm{p}<0,002)$, Brasil y Chile $(\mathrm{p}<0,02)$, Brasil y los restantes países LA $(\mathrm{p}<0,02)$, México y Chile $(\mathrm{p}<0,002)$.

La figura 2 muestra la producción anual de ECR de Argentina, Brasil, Chile, México y de los restantes países LA.

Brasil fue notoriamente el país más prolífico de ECR desde el 2000, con una tendencia a aumentar dentro del período analizado, con un $110 \%$ de aumento en el número de artículos entre los primeros 5 años y los segundos 5 años (ANCOVA, F 19,33, p < 0,01). La tendencia de México fue similar a la de Brasil, aunque no significativo el aumento (ANCOVA, F 2,65, NS). Argenti- 
Tabla 1. Estudios controlados aleatorizados efectuados en América Latina (1996-2005), ajustados por población y producto doméstico bruto PDB*, de cada país

\begin{tabular}{lccccc}
\hline Países & $\begin{array}{c}\text { Población } \\
\text { (millones) }\end{array}$ & $\begin{array}{c}\text { PDB } \\
\text { (millón US\$) }\end{array}$ & $\begin{array}{c}\text { No } \\
\text { artículos } \\
\text { ERC }\end{array}$ & $\begin{array}{c}\text { Artículos ECR/ } \\
\text { millón habitantes }\end{array}$ & $\begin{array}{c}\text { Artículos ECR/ } \\
\text { millón US\$ PDB }\end{array}$ \\
\hline Argentina & 38,7 & 183,3 & 39 & 1,008 & 0,213 \\
Bolivia & 9,9 & 9,3 & 1 & 0,101 & 0,108 \\
Brasil & 186,4 & 794,0 & 146 & 0,783 & 0,184 \\
Chile & 16,3 & 115,2 & 55 & 3,374 & 0,477 \\
Colombia & 45,6 & 45,6 & 9 & 0,197 & 0,197 \\
Costa Rica & 4,3 & 19,4 & 6 & 1,395 & 0,309 \\
Cuba & 11,3 & & 4 & 0,354 & 0,193 \\
Ecuador & 13,2 & 36,2 & 7 & 0,530 & 0,000 \\
El Salvador & 6,8 & 16,9 & 0 & 0,000 & 0,253 \\
Guatemala & 12,6 & 31,6 & 8 & 0,635 & 0,000 \\
Honduras & 7,2 & 7,9 & 0 & 0,000 & 0,112 \\
México & 103 & 868,4 & 0,835 & 0,204 \\
Nicaragua & 5,4 & 4,9 & 1 & 0,185 & 0,260 \\
Panamá & 3,2 & 15,4 & 4 & 1,250 & 0,123 \\
Paraguay & 6,2 & 8,1 & 0,161 & 0,268 \\
Perú & 27,9 & 78,4 & 21 & 0,753 & 0,035 \\
Rep. Dominicana & 8,9 & 28,3 & 1 & 0,112 & 0,059 \\
Uruguay & 3,4 & 16,9 & 1 & 0,294 & 0,072 \\
Venezuela & 26,5 & 138,8 & 10 & 0,377 & 0,175 \\
LA - 4** & 344,5 & 1861,0 & 326 & 0,946 & 0,168 \\
LA - 19 & 2387,0 & 400 & 13,906 & 0,298 \\
New York & 19,2 & 897,0 & 267 & &
\end{tabular}

*PDB: Producto doméstico bruto; ** LA4: Brasil + México + Chile + Argentina;

*** LA-19: todos los países de América Latina

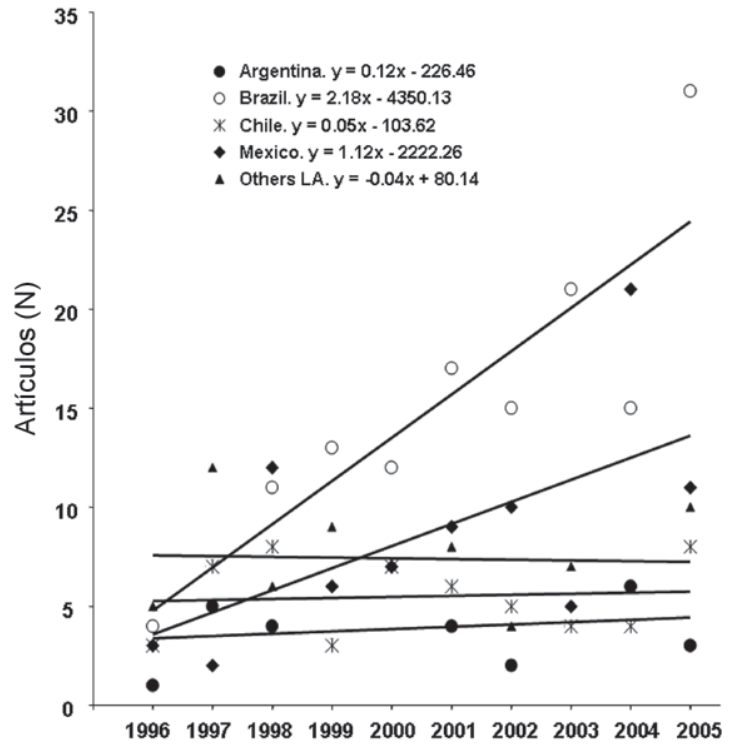

Figura 2. Correlación y regresión linear de publicaciones de estudios controlados aleatoreos/año entre 1996 y 2005 en países de América Latina seleccionados (Otros significa: los restantes países latinoamericanos). na, Chile y los restantes países de LA no mostraron un aumento de ECR en la década analizada. New York tuvo un aumento leve de ECR en el período.

\section{Ajuste por población y producto doméstico bruto}

El número de ECR para Brasil, México, Chile y Argentina como un grupo y ajustado por población, fue 2,5 veces mayor que el de los restantes países de LA. La productividad de ECR del estado de New York también ajustado por población, fue 20 veces mayor que para LA (tabla 1).

\section{Áreas temáticas Pediátricas}

Las áreas temáticas que más aportaron a los ECR fueron: nutrición (16,0\%), broncopulmonar $(13,5 \%)$, dental $(9,5 \%)$, vacunas $(8,9 \%)$ y neonatología $(8,0 \%)$. Brasil contribuyó con la mayoría de las publicaciones dentales.

En comparación, el mayor porcentaje de 
artículos publicados por científicos del estado de New York fue en las áreas de prevención de salud y psiquiatría/psicología $(23,6 \%)$ (figura 3).

Cerca del $85 \%$ de los ECR de Brasil, Chile y New York fueron realizados por equipos científicos pertenecientes a Universidades, mientras que en Argentina y México, la mayor parte se efectuaron por equipos de hospitales (51\%) o por organizaciones públicas o privadas $(23 \%$ y $14 \%$, respectivamente).

Sobre el 90\% de los ECR de Latino América se publicaron en revistas científicas internacionales con índice de impacto ISI, principalmente de USA; el restante porcentaje fue publicado en revistas locales, sin índice de impacto. La excepción fue México con un $21 \%$ de sus trabajos publicados en revistas locales con índice de impacto.

\section{Discusión}

Los resultados de nuestro estudio muestran que los ECR publicados en Latino América están distribuidos desigualmente entre los países, con una alta proporción de ellos concentrados en unos pocos países y una proporción menor o ausencia de publicaciones en los restantes. Más del $80 \%$ de las publicaciones se produjeron en 4 países: Brasil, México, Chile y Argentina. Analizados como un todo, el número de ECR aumentó a través de la década analizada.

Brasil fue notoriamente el país más prolífico de ECR en Latino América y con una clara tendencia a aumentar su producción en estos años. Estos resultados son consistentes con publicaciones previas que sitúan a Brasil entre los 20 países en el mundo con mayor producción científica en el área de la Salud y concordante con sus políticas nacionales de financiamiento científico en las últimas décadas ${ }^{4}$.

Los números absolutos de publicaciones colocan a Brasil y México como los más producti-

vos en ECR en Latino América ${ }^{15}$; sin embargo, si se ajustan las cifras por algún indicador disponible de cada país, Chile y Argentina pasan a ser los más productivos considerando su población total.

Los tópicos principales de investigación pediátrica variaron al comparar LA con el estado de New York. En LA los tópicos más frecuentes fueron: nutrición, vacunas, enfermedades respiratorias, enfermedades infecciosas y neonatología. En el estado de New York fue más dispersa le tendencia, siendo los más frecuentes: promoción/prevención de salud, psiquiatría/psicología, salud pública y adolescencia.

A pesar que algunos países no tienen ningún plan de desarrollo para financiar o establecer focos de investigación locales, los temas más estudiados reflejan que se están analizando los temas más prevalentes en la región: nutrición, enfermedades respiratorias, enfermedades infecciosas. Para la situación de las vacunas, unido a los equipos investigadores locales es importante la participación de la industria farmacéutica que financia estos estudios de alto costo.

La mayor proporción de los ECR publicados fueron efectuados por profesionales de Univer- 
sidades. Aunque en años recientes están irrumpiendo una cantidad importante de nuevas Universidades privadas, casi todos los artículos publicados fueron efectuados por equipos de universidades altamente reconocidas en la región, donde la investigación científica es una de sus tareas importantes.

Es posible que algunos ECR efectuados en este período no hayan sido publicados finalmente ${ }^{16}$. Nuestro análisis además de la base de datos PUB MED, incluyó la base LILACS, lo que nos permitió encontrar cerca de un $10 \%$ de trabajos ECR publicados en revistas no indexadas en ISI. No era el objeto de este análisis entrar a evaluar la calidad de cada ECR.

Es muy reconocido el hecho que las publicaciones científicas en Salud están altamente concentradas en los países desarrollados ${ }^{3}$. Estados Unidos de Norteamérica es el país con la mayor producción mundial, contribuyendo con más de un tercio de las publicaciones en revistas con índice de impacto ISI ${ }^{4}$.

Un reciente análisis de las tendencias en publicaciones en Salud, mostraba que ella estaba asociada a grupos de países por nivel de ingresos. Los países con altos ingresos tenían una participación relativamente constante en las publicaciones mundiales, en contraste con las de países con ingresos medio o medio-alto (ej. Argentina, México, Chile) y con países con ingresos medio-bajos (ej. Brasil), en los cuales estaba aumentando su participación (Brasil era el país más representativo de esta tendencia). Los países con bajos ingresos, principalmente de África, estaban declinando en alrededor de un $10 \%{ }^{4}$.

De acuerdo con el estudio de Science Watch, Latino América está lentamente aumentando su presencia en la investigación científica, con el aporte principalmente de Brasil, México, Argentina y Chile?

En nuestra investigación, la producción del estado de New York, USA, fue cercana a la de toda LA, incluso con un leve aumento en la década analizada. Representa una cultura de la investigación científica, con organizaciones científicas universitarias estables, gran cantidad de fondos para investigación, profesionales con una alta formación en investigación científica (programas de doctorado).
Estos resultados proporcionan evidencia adicional de la gran y creciente brecha en la producción científica en salud y en especial en Pediatría.

Actualmente, sólo un 10\% de la investigación se efectúa en países en vías de desarrollo $\mathrm{y}$ muchos de estos estudios son financiados y en general dirigidos por equipos de investigación provenientes de países desarrollados ${ }^{3}$. Muchas de estas investigaciones se efectúan en esos países por la mayor prevalencia de diversas enfermedades en ellos, pero también pueden influir los menores costos y las menores limitaciones éticas.

Hay muchas dificultades para desarrollar y para publicar en revistas internacionales, la investigación efectuada por equipos científicos de países latinoamericanos. La formación en universidades y hospitales se orienta casi exclusivamente al área clínica; los docentes no tienen en general formación en ciencia aplicada a la clínica; no hay fondos o son escasos para investigación en la mayoría de los países; no hay comunidades locales de pares científicos en Salud; hay un mal manejo del idioma inglés ${ }^{3}$.

A pesar de estas barreras, comienza a desarrollarse una conciencia de la necesidad de construir una capacidad local en LA de investigación científica como mecanismo de desarrollo del país. Está jugando un rol importante en este cambio el acceso a la información vía Internet ${ }^{3}$.

No son claras las causas que explican por qué muchos países latinoamericanos no tienen investigación científica en Pediatría de este tipo o ella es muy baja. Los ingresos económicos de esos países no explican totalmente esta escasez. Puede influir una incapacidad local política de percibir los beneficios de estas actividades y de adaptar sus estructuras para efectuar investigación científica e incorporar sus resultados.

Concluimos que hay una baja cantidad de publicaciones con estudios controlados aleatorizados en Latino América, en comparación con el estado de New York, USA. Algunos países con algún grado mayor de desarrollo están en proceso de promover y aumentar su investigación científica de calidad. Se requieren estrategias para aumentar este tipo de investigación de alta calidad. 


\section{Referencias}

1.- Sheikh A, Smeeth L, Ashcroft R: Randomized controlled trials in primary care: scope and application. Br J Gen Pract 2002; 482: 746-51.

2.- Sackett DL, Straus SE, Richardson WS, Rosenberg W, Haynes RB: Evidence-based Medicine: How to Practice and Teach EBM, 2 ${ }^{\text {nd }}$ Ed. London: Churchill Livingstone, 2000; p: 105-9.

3.- Smith R: Publishing research from developing countries. Stat Med 2002; 21: 2869-77.

4.- Paraje G, Sadana R, Karam G: Public health. Increasing international gaps in health-related publications. Science 2005; 308: 959-60.

5.- Soteriades ES, Falagas ME: Comparison of amount of biomedical research originating from the European Union and the United States. BMJ 2005; 331: 192-4.

6.- Philipson L: Medical research activities, funding, and creativity in Europe: comparison with research in the United States. JAMA 2005; 294: 1394-8.

7.- Science Watch [serial on the Internet]. 2001 Latin America: a growing presence. [cited 2005 Sept 11]; 12 (5): 1-2. Available from: http://www.sciencewatch. com/sep-oct2001/sw_sep-oct2001_page1.htm.

8.- Vergidis PI, Karavasiou AI, Paraschakis K, Bliziotis I, Falagas ME: A bibliometric analysis of global trends of research productivity in microbiology. Eur J Clin Microbiol Infect Dis 2005; 24: 342-6.
9.- Rosmarakis ES, Vergidis PI, Soteriades ES, Paraschakis K, Papastamatakipa C, Falagas ME: Estimates of global production in cardiovascular diseases research. Int J Cardiol 2005; 100: 443-9.

10.- Garfield E: Quantitative analysis of the scientific literature and its implications for science policymaking in Latin America and the Caribbean. Bull Pan Am Health Org 1995; 29: 87-95.

11.- Spinak E: Los análisis cuantitativos de la literatura científica y su validez para juzgar la producción latinoamericana. Bol Ofic Sanit Panam 1996; 120: 139-47.

12.- Pellegrini Filho A, Goldbaum M, Silvi J: Production of scientific articles on health in six Latin American countries, 1973-1992. Rev Panam Salud Publ 1997; 2: 21-32.

13.- Castillo CD, Espinoza J: Estudios clínicos avanzados en la investigación Pediátrica en Chile. 1994-2003. Rev Chil Pediatr 2005; 76: 139-42.

14.- World Bank: Data and Research. 2006. Available from: http://web.worldbank.org

15.- Hill DL: Latin America shows rapid rise in S\&E Articles. National Science Foundation, Division of Science Resources Studies 2004.

16.- Klassen TP, Wiebe N, Russell K, Stevens K, Hartling L, Craig WR, et al: Abstracts of randomized controlled trials presented at the society for pediatric research: an example of publication bias. Arch Pediatr Adolesc Med 2002; 156: 474-9. 